\title{
A rapid, high-volume cervical screening project using self-sampling and isothermal PCR HPV testing
}

Andrew Goldstein ${ }^{*}$, Yang Lei ${ }^{2}$, Lena Goldstein ${ }^{3}$, Amelia Goldstein ${ }^{4}$, Qiao Xu Bai ${ }^{5}$, Juan Felix ${ }^{6}$, Roberta Lipson ${ }^{5}$, Maria Demarco ${ }^{7}$, Mark Schiffman ${ }^{7}$ D, Didem Egemen ${ }^{7}$, Kanan T. Desai $^{7}$, Sarah Bedell ${ }^{8}$, Janet Gersten ${ }^{8}$,

Gail Goldstein ${ }^{9}$, Karen O'Keefe ${ }^{10}$, Casey O'Keefe ${ }^{11}$, Tierney O'Keefe ${ }^{12}$, Cathy Sebag ${ }^{13}$, Lior Lobel ${ }^{13}$, Anna Zhao ${ }^{5}$ and Yan Ling Lu ${ }^{5}$

\begin{abstract}
Objective: Rapid, high-volume screening programs are needed as part of cervical cancer prevention in China.

Methods: In a 5-day screening project in Inner Mongolia, 3345 women volunteered following a community awareness campaign, and self-swabbed to permit rapid HPV testing. Two AmpFire ${ }^{\mathrm{TM}}$ HPV detection systems (Atila Biosystems) were sufficient to provide pooled 15-HPV type data within an hour. HPV+ patients had same-day digital colposcopy (DC) performed by 1 of 6 physicians, using the EVA ${ }^{\mathrm{TM}}$ system (MobileODT). Digital images were obtained and, after biopsy of suspected lesions for later confirmatory diagnosis, women were treated immediately based on colposcopic impression. Suspected low- grade lesions were offered treatment with thermal ablation (Wisap), and suspected high-grade lesions were treated with LLETZ.

Results: Of 3345 women screened, 624 (18.7\%) were HPV+. Of these, 88.5\% HPV+ women underwent same-day colposcopy and 78 were treated. Later consensus histology results obtained on 197 women indicated 20 CIN2t, of whom 15 were detected and treated/referred at screening (10 by thermal ablation, 4 by LLETZ, 1 by referral).

Conclusions: Global control of cervical cancer will require both vaccination and screening of a huge number of women. This study illustrates a cervical screening strategy that can be used to screen-and-treat large numbers of women. HPV self-sampling facilitates high-volume screening. Specimens can be tested rapidly, promoting minimal loss-to-follow-up. Specifically, the AmpFire ${ }^{\mathrm{TM}}$ system used in this study is highly portable, simple, rapid (92 specimens per 65 min per unit), and economical. Visual triage can be performed on HPV+ women with a portable digital colposcope that provides magnification, lighting, and a recorded image. Diagnosis and appropriate treatment remain the most subjective elements. The digital image is under study for deep-learning based automated evaluation that could assist the management decision, either by itself or combined with HPV typing.
\end{abstract}

\footnotetext{
* Correspondence: obstetrics@yahoo.com

${ }^{1}$ Centers for Vulvovaginal Disease, Washington, DC, USA

Full list of author information is available at the end of the article
}

(C) The Author(s). 2020 Open Access This article is licensed under a Creative Commons Attribution 4.0 International License, which permits use, sharing, adaptation, distribution and reproduction in any medium or format, as long as you give appropriate credit to the original author(s) and the source, provide a link to the Creative Commons licence, and indicate if changes were made. The images or other third party material in this article are included in the article's Creative Commons licence, unless indicated otherwise in a credit line to the material. If material is not included in the article's Creative Commons licence and your intended use is not permitted by statutory regulation or exceeds the permitted use, you will need to obtain permission directly from the copyright holder. To view a copy of this licence, visit http://creativecommons.org/licenses/by/4.0/ The Creative Commons Public Domain Dedication waiver (http://creativecommons.org/publicdomain/zero/1.0/) applies to the data made available in this article, unless otherwise stated in a credit line to the data. 


\section{Introduction}

Infection with human papillomavirus (HPV) is a leading cause of cancer among women worldwide with approximately 500,000 new cervical cancer cases and 250,000 deaths each year [1]. Cervical cancer is caused by persistent infection with a group of carcinogenic HPV genotypes (HPV16, 18, 31, 33, 35, 39, 45, 51, 52, 56, 58, 59, and probably HPV68) [2]. The importance of cervical cancer is accentuated by the relatively young average age at incidence and death.

Cervical cancer screening strategies have evolved from cytology-based to HPV-based [3]. Following the identification of HPV as the cause of cervical cancer and the development of sensitive HPV tests, HPV-based screening permits the extension of screening intervals and increased impact per number of lifetime screens [3].

Without compromising yield of disease, selfsampling can increase participation and reach of cervical cancer screening programs. Self-sampling is comparable to clinician-obtained sampling for HPVbased screening, is well accepted in many populations, and has been incorporated into screening programs to improve coverage $[4,5]$.

Currently, there are no national cervical cancer screening programs in China and only $10-30 \%$ of Chinese women report having ever had cervical cancer screening [6]. Although incidence and mortality rates (15.3/100, 000 and 4.6/100,000, respectively) are moderately high, the population is so large that every year, there are approximately 100,000 new cervical cancer cases and 30, 000 deaths in China [7]. In general, women living in rural areas are less likely to report ever having had cervical cancer screening and mortality rates from cervical cancer are up to $48 \%$ higher $[6,8-10]$.

The Inner Mongolia Minority Autonomous Region (Inner Mongolia), is a vast territory that stretches in a great crescent for 1500 miles across northern China. Inner Mongolia was part of the ancient Silk Road region and is bordered to the north by Mongolia and Russia. Inner Mongolia is a geographically diverse and relatively underdeveloped province, with a population of over 24 million in the 2010 census. Forty-nine ethnic groups live in Inner Mongolia though the majority are Mongolian or Han. Additional ethnicities include Manchu, Hui, Daur, Ewenki, Oroqen, and Korean. More than 58\% of the population lives in rural areas.

Due to the level of development, complex geography, and dispersed population across the rural parts of the majority of Inner Mongolia, the conventional, multi-step screening process for cervical cancer is not feasible. The traditional process of screening with cytology, colposcopy, biopsy and subsequent treatment of women diagnosed with cervical precancer is too resource and expertise-intensive for low-income, vast regions such as
Inner Mongolia. Accordingly, techniques such as visualization with acetic acid (VIA) and HPV testing have been studied as alternative methods.

The prevalence of HPV varies amongst different ethnic and geographic regions $[11,12]$. In China, the prevalence and genotype distributions of HPV are well documented. HPV genotypes 16, 18, 52 and 58 are the most common cancer-causing types amongst Chinese women, with differing distribution rates throughout the country. The prevalence of high-risk HPV within Inner Mongolia ranges from $14.5-36.0 \%$, and this varies significantly between different ethnicities [13-15].

This study demonstrates the feasibility of a rapid, high-volume screening approach that combines selfsampled HPV testing and digital colposcopy triage to reach unscreened populations, as exemplified by Inner Mongolia, China.

\section{Methods}

This is a cross-sectional analysis using data from women attending a same-day, high-volume screening demonstration in Inner Mongolia, China. Women were contacted and, following an awareness campaign, 3345 agreed to participate and provided consent (Fig. 1). Participants were female, 30-65 years old, who provided consent to participate in the study. We excluded women who: knew or thought they might be pregnant, were unable to provide informed consent, were seriously ill, had a gross cervical mass, history of previous treatment for cervical cancer, or complete hysterectomy, or had cervical cancer screening in the past 5 years.

During a 6-day period in May 2019 that consisted of 4 clinic days and 2 travel days, 3345 Chinese women aged 30 to 65 were screened in three different medical clinics in the Inner Mongolia Minority Autonomous Region. IRB approval was obtained from United Family Hospitals Investigational Review Board, Beijing, China. Local health officials notified and registered potential participants in the preceding weeks. The vast majority of women had never been screened for cervical cancer. After obtaining informed consent, participants received a brief explanation of HPV and cervical cancer via a prerecorded video, as well as instructions on how to obtain a self-sampled vaginal specimen.

We screened 460-990 women per day (Fig. 2). The first 79 arrivals were referred directly to colposcopy after self-sampling, without awaiting the test results, to make best use of the physicians' time. This provided a subset of $69 \mathrm{HPV}$-negative referrals. Subsequently, only HPVpositive women were referred for colposcopic examination.

Self-collected vaginal specimens were collected using dry brushes and tested for 15 high-risk HPV (HPV16, HPV18, or a pool of 13 types including HPV31, 33, 35, 


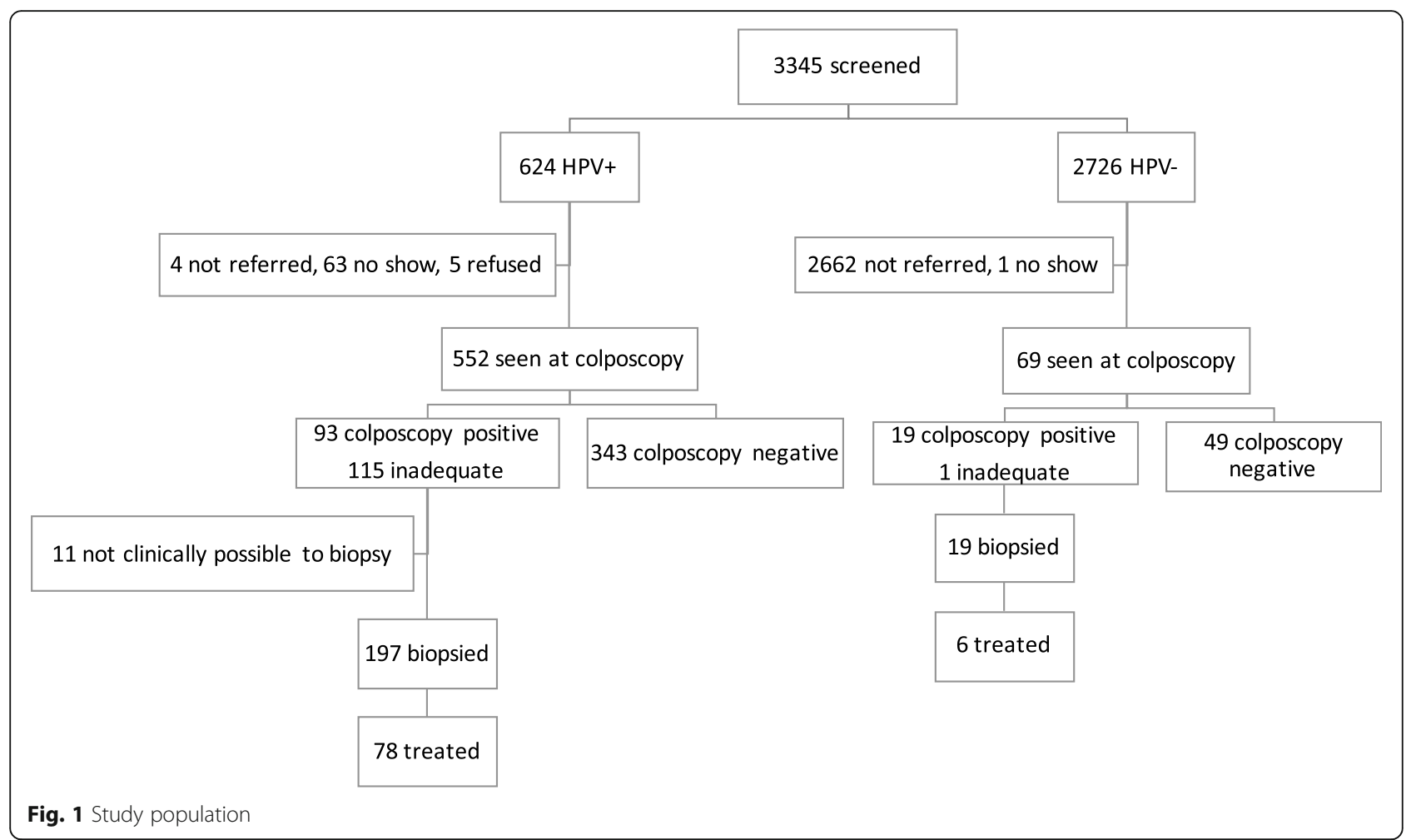

$39,45,51,52,53,56,58,59,66,68)$ on site using two AmpFire $^{\text {Tu }}$ (Atila Biosystems, California) machines running concurrently. While waiting for test results, women participated in a joint breast cancer screening effort. All women positive for high-risk HPV (hrHPV+) results were contacted via text message and returned for colposcopic examination the same day or the following day.

Digital Colposcopy (DC) was performed with the EVA system $90 \mathrm{~s}$ after the application of acetic acid. All DC was performed by one of six physicians and the EVA system was used to obtain 1-3 DC images per patient. Thin acetowhite lesions were considered suspicious for CIN1. Thick acetowhite lesions, rapidly appearing

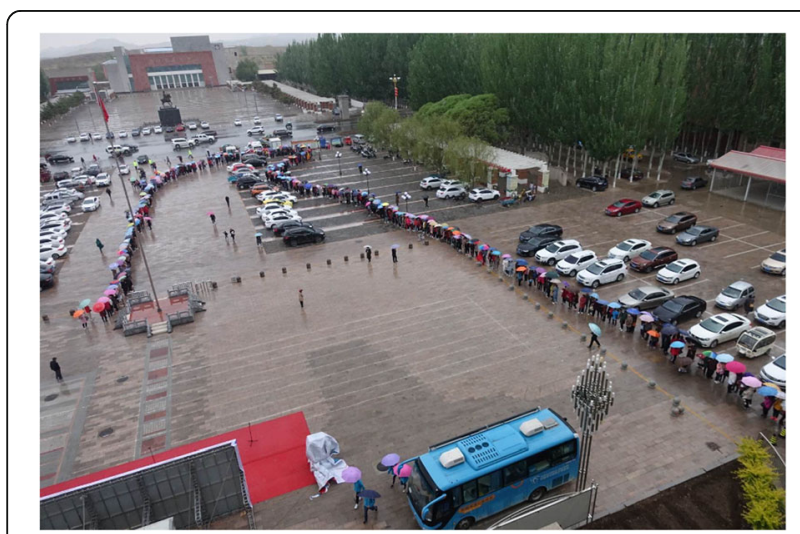

Fig. 2 Health camp approach in the Inner Mongolia Minority Autonomous Region, Photo acknowledgement: Adam Qin lesions, lesions with course mosaicism or punctuation, and lesions with sharp borders were considered suspicious for CIN2+.[27] The limitations of visual assessment for grading are acknowledged and discussed below.

If DC was positive for cervical abnormalities (suspected $\mathrm{CIN} 1+$ ), patients underwent cervical biopsy of the exocervix using a SoftBiopsy ${ }^{\text {min }}$ (Histologics, California) brush for subsequent confirmatory diagnosis, but were treated presumptively with thermocoagulation or loop electrosurgical excision procedure (LLETZ) the same day. The SoftBiopsy ${ }^{\text {ma }}$ (Histologics, California) brush obtains tissue from the entire exocervix to be processed in a single slide. Compared with multiple biopsies from biopsy forceps, it is faster to obtain tissue from multiple areas of the exocervix and it causes less trauma and bleeding, making it easier to perform thermocoagulation (if needed) immediately after biopsy.

Thermocoagulation was performed when DC findings were suspicious for CIN1 lesions using one of two different thermocoagulation systems: C3 thermo-coagulator (WISAP, Germany) or TC thermocoagulator ${ }^{\mathrm{Tw}}$ (Cure Medical, Utah). A LLETZ was performed when findings were highly suspicious for CIN2+ or when thermal ablation was not technically possible.

If no acetowhite changes were seen and the entire transformation zone was visible, the patients were informed of the findings and not immediately treated. Repeat HPV testing in 1 year was advised; although the availability of such follow-up was recognized not to be 
assured, immediate treatment in the absence of a visible lesion was judged not to be warranted. If the transformation zone was not fully visible on DC or if the lesion extended in the endocervical canal, endocervical curettage (ECC) was performed.

Overall, at colposcopy, clinician colposcopic impression led to 216 women biopsied and, of those, 84 immediately treated. Immediate treatment included thermal ablation, LLETZ, and none (including women who refused treatment and those treated later, based on histologic results). All biopsy specimens were subsequently processed, and read twice, by two pathologists (QXB, JF). The original histopathologic diagnoses included CIN1, CIN1-2, CIN2, CIN2-3, CIN3, and inadequate. For this research analysis, precancer case status among the HPV-positive women was defined as follows: CIN3 by either pathologist or CIN2 by both pathologists. The remaining adequate samples were considered $<$ CIN2.

The few women with unanticipated high-grade lesions $(\mathrm{CIN} 2+)$ were later contacted with their histology results and were counseled to follow up at the regional hospital for appropriate management. As mentioned, while women were waiting for the results of their HPV tests, they were screened for breast cancer with a portable ultrasound unit (results reported separately). Throughout the screening and treatment process, for educational purposes, local doctors and nurses were taught colposcopy techniques by the visiting physicians and received training to evaluate DC images.

\section{Results}

Using self-sampled HPV testing, 3345 women were screened. Most women screened were ethnically Han (64.4\%) and Meng (20.1\%) (Table 1). The majority (63.7\%) were ages $35-49$.

HPV-positive women $(624,18.7 \%)$ were invited for colposcopy and 552 attended the medical examination. Colposcopic impression was inadequate for $20.8 \%$. Of the rest, $78.5 \%$ impressions were normal and not requiring biopsy.

The characteristics of the 197 HPV-positive women that underwent colposcopy and had a biopsy are listed in Table 2. Biopsies were collected with SoftBiopsy ${ }^{\circ}$ brush (44.2\%), ECC (52.8\%), or both (3\%).

From the 78 women who received treatment, 71 $(89.7 \%)$ were treated with thermal ablation and 7 (9.0\%) with LLETZ.

This rapid, high-volume program yielded $0.60 \%$ of precancer (20 out of 3345) in the screening population. From the 20 cases identified by histology, 10 were infected with HPV16, 1 with HPV18, and 8 with other types (HPV status was unknown for 1 case) (Table 3 ). Thus, we observed the expected increased risk associated with HPV16 and HPV18. Fifteen of the 20 HPV-positive
Table 1 General information of the screened residences in three areas in Inner Mongolia

\begin{tabular}{lllll}
\hline & Areas & & & \\
\cline { 2 - 5 } & Balinyouqi & Aershan & Molidawa & Total \\
\hline Age & & & & \\
$30 \sim 34$ & $76(8.3)$ & $5(2.6)$ & $55(5.5)$ & $136(6.4)$ \\
$35 \sim 39$ & $143(15.7)$ & $15(7.8)$ & $235(23.4)$ & $393(18.5)$ \\
$40 \sim 44$ & $157(17.2)$ & $32(16.6)$ & $237(23.6)$ & $426(20)$ \\
$45 \sim 49$ & $222(24.3)$ & $46(23.8)$ & $268(26.7)$ & $536(25.2)$ \\
$50 \sim 54$ & $153(16.8)$ & $60(31.1)$ & $152(15.1)$ & $365(17.2)$ \\
$55 \sim 59$ & $116(12.7)$ & $31(16.1)$ & $44(4.4)$ & $191(9)$ \\
$60 \sim 64$ & $45(4.9)$ & $4(2.1)$ & $14(1.4)$ & $63(3)$ \\
Mean & $46.2 \pm 8.0$ & $48.4 \pm 6.2$ & $44.0 \pm 6.7$ & $45.4 \pm 7.4$ \\
Ethnicity & & & & \\
Han & $540(59.1)$ & $148(75.1)$ & $681(67)$ & $1369(64.4)$ \\
Meng & $344(37.7)$ & $39(19.8)$ & $44(4.3)$ & $427(20.1)$ \\
Da & $0(0)$ & $0(0)$ & $189(18.6)$ & $189(8.5)$ \\
unknown & $29(3.2)$ & $10(5.1)$ & $103(10.1)$ & $139(6.3)$ \\
Parity & & & & \\
0 & $4(0.4)$ & $3(1.6)$ & $13(1.3)$ & $20(0.9)$ \\
1 & $393(43.6)$ & $157(84)$ & $553(55)$ & $1103(51.9)$ \\
2 & $442(49.1)$ & $22(11.8)$ & $376(37.4)$ & $840(39.5)$ \\
$\geq 3$ & $60(6.7)$ & $5(2.6)$ & $63(6.3)$ & $128(6.1)$ \\
unknown & $2(0.2)$ & $0(0)$ & $0(0)$ & $2(0.1)$ \\
\hline
\end{tabular}

cases received treatment: 10 by thermal ablation, 4 by LLETZ, 1 by referral. We notified the clinicians of the 5 untreated cases for follow-up after the program ended.

\section{Discussion}

As its main conclusion, this study showed that largescale cervical cancer screening efforts are feasible using self-sampling and rapid HPV testing. Availability of results within $2 \mathrm{~h}$ of collection made it possible for a single-visit screen and treat program.

Among the $20 \mathrm{CIN} 2+$ cases, we saw the expected strong relationship between HPV positivity and presence of precancer, with some insights on partial HPV genotyping. However, our evaluation of absolute sensitivity of the HPV test was imperfect, because most HPV-negative women did not receive colposcopic evaluations. HPV DNA negativity typically predicts an extremely low risk of prevalent or incipient cervical cancer (or even precancer) [16].

As the success of cervical cancer screening programs has dramatically decreased the rates of cervical cancer in developed nations, the global burden of this disease falls mainly in areas of limited resources. It is estimated that the number of 35-64-year-old women in rural China exceeds 150 million. As the vast majority of these women 
Table 2 Descriptive statistics for HPV-positive women with biopsies taken $(n=197)$

\begin{tabular}{ll}
\hline & Frequency $\mathbf{n}(\%)$ \\
\hline HPV results & $33(17.2)$ \\
$16^{\text {a }}$ & $16(8.3)$ \\
18 & $143(74.5)$ \\
Others & 5 \\
Colposcopy impression & \\
High & $21(10.7)$ \\
Low & $72(36.5)$ \\
Inadequate & $104(52.8)$ \\
Biopsy taken & \\
Brush & $87(44.2)$ \\
ECC & $104(52.8)$ \\
Both & $6(3.0)$ \\
Histology & \\
CIN2+ & $20(10.2)$ \\
$<$ CIN2 & $176(89.8)$ \\
Treatment & \\
Thermoablation & $70(89.7)$ \\
LLETZ & $7(9.0)$ \\
Referral & $1(1.3)$ \\
None & 119 \\
\hline Includes 1 multiple infection with HPV types 16 and 18 & \\
\hline &
\end{tabular}

have never been screened, strategies must be developed that allow for the screening and treating of large numbers of women. We demonstrate a screen-and-treat model that is low-cost, rapid, and capable of being implemented on a large scale, as evidenced by the ability to screen 3345 women in less than one week. The authors have previously described a similar strategy for cervical cancer screening in the Yunnan Province [17].

The first component of this model consists of selfobtained HPV specimens for rapid testing. It is practical

Table 3 Histology by HPV status for women with biopsies taken $(n=216)$

\begin{tabular}{llll}
\hline HPV status & \multicolumn{2}{l}{ Histology } & \\
\cline { 2 - 3 } & $\begin{array}{l}\text { CIN2+ } \\
\mathbf{n}(\mathbf{c o l} \%)\end{array}$ & $\begin{array}{l}<\mathbf{C I N 2} \\
\mathbf{n}(\mathbf{c o l} \%)\end{array}$ & $\begin{array}{l}\text { Total } \\
\mathbf{n}(\mathbf{c o l} \%)\end{array}$ \\
\hline $16+$ & $10(50.0 \%)$ & $23(11.7 \%)$ & $33^{\text {a }}(15.3 \%)$ \\
$18+$ & $1(5.0 \%)$ & $15(7.7 \%)$ & $16(7.4 \%)$ \\
Other hrHPV+ & $8(40.0 \%)$ & $135(68.9 \%)$ & $143(66.2 \%)$ \\
Unknown type & $1(5.0 \%)$ & $4(2.0 \%)$ & $5(2.3 \%)$ \\
HPV- (controls) & $0(0.0 \%)$ & $19(9.7 \%)$ & $19(8.8 \%)$ \\
Total & 20 & 196 & 216 \\
\hline
\end{tabular}

${ }^{a}$ Includes 1 multiple infection with HPV types 16 and 18 for women in China to obtain self-collected HPV specimens, which then undergo rapid testing. Self-swabbing for HPV appears to be acceptable to Chinese women, regardless of their ethnicity.

Rapid testing is a critical aspect of this model as it allows for same-day treatment. The highly portable isothermal PCR based HPV AmpFire ${ }^{\mathrm{Tm}}$ testing system takes $1 \mathrm{~h}$ to run 94 specimens and provides partial genotyping (another test kit is available for complete typing). It is minimally labor intensive and does not require a high degree of technical expertise to run. The self-swab specimens can be stored dry (without collection media) and the reagents can be stored at room temperature for several weeks. Furthermore, the price per specimen is approximately \$US 7 and the same system can be used to test for other sexually transmitted infections.

The second component of this proposed model is to perform DC on all $\mathrm{HPV}+$ women with a highly portable DC system. Our program included 6 physicians, a level of expert involvement that is often not available. Given the huge number of women in China who have never been screened as compared to the number of physicians available, DC is a modality that could be performed (if authorized) by mid-level providers such as nurses or midwives. Digital images obtained have excellent resolution and areas of question can be magnified for better interpretation. In addition, captured images can be used for continued education of mid-level providers, and the images can be sent electronically, through a secure, cloud-based portal, to an expert colposcopist for consultation of difficult cases.

Our study supports assistive technology to aid clinical decision making based on human visual impression. Even when performed by experienced specialists, colposcopic impression shows subjectivity and limited reliability or inter-observer agreement [18]. In addition to its inherent subjectivity, it requires highly trained human resources that are not available in some settings.

Ongoing efforts using Automated Evaluation (AVE) of the cervix show promising results using archived images from a consortium of sites [19, 20]. AVE has been evaluated for cervical cancer screening in the general population and as triage of HPV positive tests. When used as an aid to VIA (possibly in combination with HPV type), it might improve risk stratification of screening and triage, minimizing the subjectivity of human visual interpretation.

The third component of this screen-and-treat model is to use the clinical impression obtained from the DC images, perhaps assisted by AVE, to determine immediate and appropriate treatment. Thermocoagulation is inexpensive, highly portable, and a highly effective treatment modality for cervical intraepithelial lesions caused by HPV. 
Cervical cancer screening programs could be combined with same-day vaccination efforts for HPV and other medical interventions. For example, we combined cervical and breast screening as part of the same program.

The screening model proven to be feasible by this effort is promising, but might not be widely applicable at the present time. The health camp approach (Fig. 2) is, by its nature, crowded and might be contraindicated until social gathering is again safe. Where social distancing remains a priority due to COVID-19, cervical cancer prevention efforts will need to be adjusted accordingly to maintain a net benefit for participants, as discussed by Ajenifuja et al. in a companion article. Portability of this screening model in terms of feasibility, acceptability, yield, sustainability, overall cost-effectiveness, and long-term impact also needs to be demonstrated in diverse geographic settings.

\section{Acknowledgements}

Photo acknowledgement: Adam Qin.

\section{Authors' contributions}

All authors contributed to planning and conduct of screening project, except the $\mathrm{NCl}$ authors contributed to data analysis and composition of manuscript with Dr. A. Golstein. The author (s) read and approved the final manuscript.

\section{Funding}

This work was supported by the Gynecologic Cancers Research Foundation, the United Foundation for China's Health, and from regional health offices of the Peoples Republic of China.

\section{Availability of data and materials}

Data available upon reasonable request from corresponding author Dr. A. Goldstein.

\section{Ethics approval and consent to participate}

IRB approval was obtained prior the performance of the study from the United Family Hospitals' Investigational review board. Approval number UFHIRB: E2019-003.

\section{Consent for publication}

The authors consent to publish.

\section{Competing interests}

Andrew Goldstein: President of the Gynecologic Cancers Research Foundation: a non-profit 501 (c)3 corporation. Received research funding from the Gynecologic Cancers Research Foundation.

Yang Lei: none to report.

Lena Goldstein: none to report.

Amelia Goldstein: none to report.

Qiao Xu Bai: none to report.

Juan Felix: none to report.

Roberta Lipson: Director, United Foundation for China's Health, a non-profit 501 (c)3 corporation. Shareholder in MobileODT.

Maria Demarco, Mark Schiffman, Didem Egemen, Kanan Desai: NCI has received cervical screening supplies and results at no cost from several companies (Roche, BD, Qiagen, MobileODT) for independent evaluations of test performance.

Sarah Bedell: none to report.

Janet Gersten: none to report.

Gail Goldstein: Is a board member of the Gynecologic Cancers Research

Foundation: a non-profit 501 (c)3 corporation.

Karen O'Keefe: none to report.

Casey O'Keefe: none to report.
Tierney O'Keefe: none to report.

Cathy Sebag: Was an employee of MobileODT during the data collection. Lior Lobel: Was an employee of MobileODT during the data collection. Anna Zhao: employee, United Foundation for China's Health, a non-profit 501(c)3 corporation.

Yan Ling Lu: none to report.

\section{Author details}

${ }^{1}$ Centers for Vulvovaginal Disease, Washington, DC, USA. ${ }^{2}$ Peking University Cancer Hospital, Beijing, China. ${ }^{3}$ Yale University, New Haven, CT, USA. ${ }^{4}$ Duke University, Durham, NC, USA. ${ }^{5}$ United Family Hospitals, Beijing, China. ${ }^{6}$ Medical College of Wisconsin, Milwaukee, WI, USA. ${ }^{7}$ Division of Cancer Epidemiology and Genetics, National Cancer Institute, National Institutes of Health, Rockville, MD, USA. ${ }^{8}$ New Age Women's Health, Miami, Florida, USA. ${ }^{9}$ Annapolis Dermatology Center, Annapolis, MD, USA. ${ }^{10}$ Bellingham Bay Family Medicine, Bellingham, WA, USA. ${ }^{11}$ Pacific Northwest Urology Specialists, Bellingham, WA, USA. ${ }^{12}$ Scripps College, Claremont, California, USA. ${ }^{13}$ MobileODT, Tel Aviv, Israel.

Received: 29 July 2020 Accepted: 29 September 2020

Published online: 22 October 2020

\section{References}

1. (IARC) IAfRoC. Globocan 2012: Estimated Cancer Incidence, Mortality and Prevalence Worldwide in 2012. 2016. http://globocan.iarc.fr/Pages/fact_ sheets_cancer.aspx (accessed 01/01/2019).

2. Schiffman M, Castle PE, Jeronimo J, Rodriguez AC, Wacholder S. Human papillomavirus and cervical cancer. Lancet. 2007;370(9590):890-907.

3. Schiffman M, Wentzensen N. Human papillomavirus infection and the multistage carcinogenesis of cervical cancer. Cancer Epidemiol Biomark Prev. 2013;22(4):553-60.

4. Arbyn M, Castle PE. Offering self-sampling kits for HPV testing to reach women who do not attend in the regular cervical Cancer screening program. Cancer Epidemiol Biomark Prev. 2015;24(5):769-72.

5. Belinson JL, Du H, Yang B, et al. Improved sensitivity of vaginal selfcollection and high-risk human papillomavirus testing. Int J Cancer. 2012; 130(8):1855-60

6. Mo X, Gai Tobe R, Wang L, et al. Cost-effectiveness analysis of different types of human papillomavirus vaccination combined with a cervical cancer screening program in mainland China. BMC Infect Dis. 2017;17(1):502.

7. Duan R, Qiao Y, Clifford G, Zhao F. Cancer burden attributable to human papillomavirus infection by sex, cancer site, age, and geographical area in China. Cancer Med. 2020;9(1):374-84.

8. Huang S, Afonina I, Miller BA, Beckmann AM. Human papillomavirus types 52 and 58 are prevalent in cervical cancers from Chinese women. Int J Cancer. 1997:70(4):408-11.

9. Lo KW, Wong YF, Chan MK, et al. Prevalence of human papillomavirus in cervical cancer: a multicenter study in China. Int J Cancer. 2002; 100(3):327-31

10. Liu J, Rose B, Huang $X$, et al. Comparative analysis of characteristics of women with cervical cancer in high- versus low-incidence regions. Gynecol Oncol. 2004;94(3):803-10.

11. Bruni L, Diaz M, Castellsagué X, Ferrer E, Bosch FX, de Sanjosé S. Cervical human papillomavirus prevalence in 5 continents: meta-analysis of 1 million women with normal cytological findings. J Infect Dis. 2010;202(12):1789-99.

12. Forman D, de Martel C, Lacey CJ, et al. Global burden of human papillomavirus and related diseases. Vaccine. 2012;30(Suppl 5):F12-23.

13. Wang $X, J i$ Y, Li J, et al. Prevalence of human papillomavirus infection in women in the autonomous region of Inner Mongolia: a population-based study of a Chinese ethnic minority. J Med Virol. 2018;90(1):148-56.

14. Wang $M Z$, Feng RM, Wang $S$, et al. Clinical performance of human papillomavirus testing and visual inspection with acetic acid in primary, combination, and sequential cervical Cancer screening in China. Sex Transm Dis. 2019;46(8):540-7.

15. Ji Y, Ma XX, Li Z, Peppelenbosch MP, Ma Z, Pan Q. The burden of human papillomavirus and chlamydia trachomatis Coinfection in women: a large cohort study in Inner Mongolia, China. J Infect Dis. 2019;219(2):206-14.

16. Gage JC, Schiffman M, Katki HA, et al. Reassurance against future risk of precancer and cancer conferred by a negative human papillomavirus test. J Natl Cancer Inst. 2014;106(8):dju153. https://doi.org/10.1093/jnci/dju153. Print 2014 Aug. PMID: 25038467. 
17. Goldstein A, Goldstein LS, Lipson R, et al. Assessing the feasibility of a rapid, high-volume cervical cancer screening programme using HPV self-sampling and digital colposcopy in rural regions of Yunnan, China. BMJ Open. 2020; 10(3): $: 035153$.

18. Massad LS, Jeronimo J, Schiffman M. National institutes of health/American Society for C, cervical pathology research $\mathrm{G}$. Interobserver agreement in the assessment of components of colposcopic grading. Obstet Gynecol. 2008; 111(6):1279-84.

19. Xue Z, Novetsky AP, Einstein MH, Marcus JZ, Befano B, Guo P, Demarco M, Wentzensen N, Long LR, Schiffman M, Antani S. A demonstration of automated visual evaluation of cervical images taken with a smartphone camera. Int J Cancer. 2020. https://doi.org/10.1002/ijc.33029. Epub ahead of print. PMID: 32356305

20. Hu L, Bell D, Antani S, et al. An observational study of deep learning and automated evaluation of cervical images for Cancer screening. J Natl Cancer Inst. 2019;111(9):923-32.

\section{Publisher's Note}

Springer Nature remains neutral with regard to jurisdictional claims in published maps and institutional affiliations.

Ready to submit your research? Choose BMC and benefit from:

- fast, convenient online submission

- thorough peer review by experienced researchers in your field

- rapid publication on acceptance

- support for research data, including large and complex data types

- gold Open Access which fosters wider collaboration and increased citations

- maximum visibility for your research: over $100 \mathrm{M}$ website views per year

At $\mathrm{BMC}$, research is always in progress.

Learn more biomedcentral.com/submissions 